\title{
Strategies for coupling global and limited-area ensemble Kalman filter assimilation
}

\author{
D. Merkova ${ }^{1}$, I. Szunyogh ${ }^{2}$, and E. Ott ${ }^{1}$ \\ ${ }^{1}$ University of Maryland, College Park, MD, USA \\ ${ }^{2}$ Texas A\&M, College Station, TX, USA
}

Received: 4 August 2010 - Revised: 7 February 2011 - Accepted: 17 May 2011 - Published: 27 June 2011

\begin{abstract}
This paper compares the forecast performance of four strategies for coupling global and limited area data assimilation: three strategies propagate information from the global to the limited area process, while the fourth strategy feeds back information from the limited area to the global process. All four strategies are formulated in the Local Ensemble Transform Kalman Filter (LETKF) framework.

Numerical experiments are carried out with the model component of the National Centers for Environmental Prediction (NCEP) Global Forecast System (GFS) and the NCEP Regional Spectral Model (RSM). The limited area domain is an extended North-America region that includes part of the north-east Pacific. The GFS is integrated at horizontal resolution T62 (about $150 \mathrm{~km}$ in the mid-latitudes), while the RSM is integrated at horizontal resolution $48 \mathrm{~km}$. Experiments are carried out both under the perfect model hypothesis and in a realistic setting. The coupling strategies are evaluated by comparing their deterministic forecast performance at $12-\mathrm{h}$ and 48 -h lead times.

The results suggest that the limited area data assimilation system has the potential to enhance the forecasts at $12-\mathrm{h}$ lead time in the limited area domain at the synoptic and subsynoptic scales (in the global wave number range of about 10 to 40). There is a clear indication that between the forecast performance of the different coupling strategies those that cycle the limited area assimilation process produce the most accurate forecasts. In the realistic setting, at 12-h forecast time the limited area systems produce more modest improvements compared to the global system than under the perfect model hypothesis, and at 48 -h forecast time the global forecasts are more accurate than the limited area forecasts.
\end{abstract}

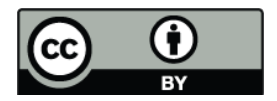

Correspondence to: D. Merkova (dagmar.merkova@nasa.gov)

\section{Introduction}

An atmospheric limited area model uses time-dependent lateral boundary conditions provided by a global atmospheric model. In current practice, the initial conditions for the limited area model are either analyses prepared using the global model and interpolated to the higher resolution grid of the limited area model, or analyses prepared by using a data assimilation system specifically designed to produce initial states for use by the limited area model. In the latter case, the analysis inside the limited area domain is obtained independently of the global analysis (e.g., Torn et al., 2006; Zhang et al., 2006; Huang et al., 2009). The aforementioned two approaches are motivated by the practical constraint that most weather prediction centers and research groups who run limited area models have access to global analysis products, but do not have the capability to produce global analyses. The only exceptions are a handful of operational NWP centers, e.g., the National Centers for Environmental Prediction (NCEP), who prepare both global and limited area analyses, but, mainly for practical reasons, follow one of the two aforementioned approaches.

In this paper, we consider the scenario in which we have access to both the global and the limited-area model and an ensemble-based data assimilation system. Our goal is to begin to address the problem of finding that configuration of the coupling between these three components of the forecast system, which provides the best global and limited area model forecasts. In particular, we compare the deterministic forecast performance of the system for different coupling strategies using both simulated and operationally used observations of the atmosphere. In our experiments, the global model is the model component of the Global Forecast System (GFS) of the National Centers for Environmental Prediction (NCEP) (Sela, 1980) integrated at a T62L28 (about

Published by Copernicus Publications on behalf of the European Geosciences Union and the American Geophysical Union. 
$150 \mathrm{~km}$ ) horizontal resolution, the limited area model is the Regional Spectral Model (RSM) of NCEP (Juang, 1992; Juang and Kanamitsu, 1994; Juang et al., 1997; Juang and Hong, 2001) integrated at $48 \mathrm{~km}$ and L28 resolution, while the data assimilation system is the Local Ensemble Transform Kalman Filter (Ott et al., 2004; Hunt et al., 2007; Szunyogh et al., 2008). We choose the NCEP RSM for this study, because it has the most consistent dynamics, among all limited area models, with that of the NCEP GFS model. In particular, the two models share the same physical parametrization packages and the GFS model solution affects the RSM solutions not only at the lateral boundaries, but also in the entire limited area domain. We design numerical experiments to start assessing the forecast value added by the limited area assimilation. We stress that the main aim of the present study is to investigate the benefits of coupling global and limited area data assimilation in principle, rather than develop recommendations for the operational practice: since the resolution of our limited area model is lower than that of the operational global model of NCEP, which at the time of writing is T574 (about $27 \mathrm{~km}$ ), the resolution in our experimentsis not sufficient to assess the quality of the current operational systems. In addition, we do not assimilate satellite radiance observations: Since global forecast systems tend to benefit more from the assimilation of past observations, including large number of satellite radiance observations assimilated at earlier analysis time, than the limited area systems, where the propagation of information from past observations is hindered by the imperfect boundary conditions (A. Lorenc, personal communication, 2011), our experiments most likely underestimate the predictive value of the global system relative to that of the limited area system.

The structure of the paper is as follows. Section 2 describes the coupling strategies we consider in this study. Section 3 explains the design of the numerical experiments that we carry out to assess the performance of the different coupling strategies. The results of the numerical experiments obtained for the perfect model scenario are presented in Sect. 4, while the results obtained with assimilating observations of the real atmosphere are reported in Sect. 5. Our conclusions are summarized in Sect. 6 .

\section{Coupling strategies}

To design strategies for the coupling of a global and a limited area data assimilation system, we assume that the higher resolution limited area model provides a more accurate representation of the atmospheric dynamics in the limited area than does the global model. Our goal is to take advantage of the availability of this presumed better model information within the limited area to improve the quality of the analyses. We introduce our strategies assuming that the data assimilation component is based on an ensemble transform algorithm (e.g., Bishop et al., 2001; Hunt et al., 2007). While Strategy 1 is a conventional uncoupled approach, which could be easily implemented using any data assimilation algorithm, and Strategy 2 and 3 could be implemented using any ensemblebased schemes, Strategy 4 takes advantage of the fact that the ensemble transform algorithm provides a straightforward way to propagate information from the limited area data assimilation process to the global process.

\subsection{Global and limited area model dynamics}

The global model dynamics $\boldsymbol{g}$, defined by

$\boldsymbol{x}_{g}\left(t_{\mathrm{f}}\right)=\boldsymbol{g}\left[\boldsymbol{x}_{g}\left(t_{\mathrm{i}}\right)\right]$,

propagates an estimate $\boldsymbol{x}_{g}(t)$ of the global atmospheric state between an initial time $t_{\mathrm{i}}$ and a final time $t_{\mathrm{f}}$. The components of $\boldsymbol{x}_{g}(t)$ are the spatially discretized atmospheric state variables (e.g., temperature, components of the wind vector, surface pressure, humidity variables, etc.). The limited area model dynamics $f$, defined by

$x_{l}\left(t_{\mathrm{f}}\right)=\boldsymbol{f}\left[\boldsymbol{x}_{l}\left(t_{\mathrm{i}}\right), \boldsymbol{x}_{g}\left(t_{\mathrm{i}}\right)\right]$,

propagates an estimate $\boldsymbol{x}_{l}(t)$ of the atmospheric state in a limited area sub-domain of the globe at a resolution that is higher than that of $\boldsymbol{x}_{g}(t)$. Our notation in Eq. (2) reflects that for the NCEP RSM, the global initial condition affects the limited area model solution both inside and at the boundaries of the limited area domain. We introduce the notation

$\boldsymbol{x}_{l}^{\prime}(t)=\boldsymbol{x}_{l}(t)-\mathcal{L}\left[\boldsymbol{x}_{g}(t)\right]$

for the difference between the high resolution and the global state estimate in the limited area domain. In Eq. (3), $\mathcal{L}$ is the mapping from the state space of the global model onto the state space of the limited area model. In practice, this mapping is an interpolation from the lower resolution grid of the global model to the higher resolution grid of the regional model. While the limited area model resolves motions at scales that are smaller than the smallest scales resolved by the global model, there are scales that contribute to both $\boldsymbol{x}_{l}(t)$ and $\boldsymbol{x}_{g}(t)$. Thus, $\boldsymbol{x}_{l}^{\prime}(t)$ is a combination of flow features resolved by both models and features resolved only by the limited area model.

\subsection{The motivation for coupled data assimilation}

The derivation of the version of the LETKF which is considered in this study is based on the assumption that a model can provide a perfect representation of the dynamics of the observed system (Ott et al., 2004; Hunt et al., 2007). An implementation of the scheme on a numerical weather prediction model inevitably violates this assumption. One particular source of the error is the spatial discretization of the dynamics: the atmospheric state at time $t$ is represented by a spatially continuous vector field $\boldsymbol{u}(t)$, while a model uses a finite-dimensional discretization $\boldsymbol{x}(t)$ of $\boldsymbol{u}(t)$ assuming that a 
suitable projection $\mathcal{P}: \boldsymbol{x}^{t}(t)=\mathcal{P}[\boldsymbol{u}]$ exists. (Here, the superscript $t$ indicates that $\boldsymbol{x}^{t}(t)$ is the model state representation of the true atmospheric state.) Thus the finite-dimensional model dynamics $\boldsymbol{g}$ and $\boldsymbol{f}$ ignore an infinite number of interactions associated with the unresolved flow components. While parametrization of the sub-grid (unresolved) processes are designed to account for the effects of the unresolved scales on the resolved scale (e.g., Kalnay, 2002), in general, a higher resolution model is expected to provide a more accurate representation of the atmospheric dynamics. The motivation for employing a limited area model is to provide a more accurate representation of the atmospheric dynamics in a limited area domain of particular interest. Our intended purpose in coupling the global and limited area data assimilation processes is to take advantage of the presumed superiority of the limited area model in the limited domain to improve the accuracy of the limited area analyses.

\subsection{Ensemble transform data assimilation schemes}

An ensemble-based data assimilation system obtains the state estimate at analysis time $t_{n}$ in two steps: (i) in the forecast step, a prior estimate of the state, called the background, and an estimate of the uncertainty in the background are obtained by propagating information from the previous analysis time $t_{n-1}$ to $t_{n}=t_{n-1}+\Delta t$ using the model dynamics; and (ii) in the state update step, the prior estimates of the state and its uncertainty are updated based on the observations collected in the time window $\left[t_{n}-\Delta t / 2, t_{n}+\Delta t / 2\right]$.

Formally, the forecast step involves preparing a K-member ensemble of background forecasts $\left\{\boldsymbol{x}^{b(k)}\left(t_{n}\right), k=1, \ldots, K\right\}$. For instance, in a global data assimilation system

$\boldsymbol{x}_{g}^{b(k)}\left(t_{n}\right)=\boldsymbol{g}\left[\mathbf{x}_{g}^{a(k)}\left(t_{n-1}\right)\right], \quad k=1, \ldots, K$,

where $\left\{\mathbf{x}_{g}^{a(k)}\left(t_{n-1}\right), k=1, \ldots, K\right\}$ are the members of the analysis ensemble at the previous analysis time $t_{n-1}$. The background state $\overline{\mathbf{x}}^{b}\left(t_{n}\right)$ is defined by the ensemble mean,

$\overline{\mathbf{x}}^{b}\left(t_{n}\right)=K^{-1} \sum_{k=1}^{K} \mathbf{x}^{b(k)}\left(t_{n}\right)$,

while the uncertainty in the estimate $\overline{\mathbf{x}}^{b}\left(t_{n}\right)$ is described by the ensemble based estimate

$\mathbf{P}^{b}\left(t_{n}\right)=(K-1)^{-1} \mathbf{X}^{b}\left(t_{n}\right)\left[\mathbf{X}^{b}\left(t_{n}\right)\right]^{T}$,

of the background error covariance matrix. Here $\mathbf{X}^{b}\left(t_{n}\right)$ is the matrix whose $k$-th column is the $k$-th background ensemble perturbation $\boldsymbol{x}^{b(k)}=\boldsymbol{x}^{b(k)}\left(t_{n}\right)-\overline{\mathbf{x}}^{b}\left(t_{n}\right)$.

In an ensemble-transform-based data assimilation scheme the ensemble mean analysis is obtained by

$\overline{\boldsymbol{x}}^{a}\left(t_{n}\right)=\overline{\boldsymbol{x}}^{b}\left(t_{n}\right)+\mathbf{X}^{b}\left(t_{n}\right) \boldsymbol{w}^{a}\left(t_{n}\right)$,

where the "weight vector" $\boldsymbol{w}^{a}\left(t_{n}\right)$ is the value of $\boldsymbol{w}$ that minimizes the quadratic cost function

$$
\begin{aligned}
J(\boldsymbol{w})= & (k-1) \boldsymbol{w}^{T} \boldsymbol{w}+ \\
& +\left(\boldsymbol{y}^{o}\left(t_{n}\right)-\boldsymbol{h}\left[\overline{\boldsymbol{x}}^{b}\left(t_{n}\right)+\mathbf{X}^{b}\left(t_{n}\right) \boldsymbol{w}\right]\right)^{T} \boldsymbol{R}^{-1}\left(t_{n}\right)\left(\boldsymbol{y}^{o}\left(t_{n}\right)\right. \\
& \left.-\boldsymbol{h}\left[\overline{\boldsymbol{x}}^{b}\left(t_{n}\right)+\mathbf{X}^{b}\left(t_{n}\right) \boldsymbol{w}\right]\right) .
\end{aligned}
$$

In the local formulation of the ensemble-transform-based scheme used in this study, the weights are slowly varying function of location (Ott et al., 2004; Hunt et al., 2007). $\boldsymbol{y}^{o}\left(t_{n}\right)$ is the vector of observations assimilated at time $t_{n}$ and the observation operator $\boldsymbol{h}(\boldsymbol{x})$ maps the model representation of the atmospheric state to observables at observation times. The observation operator is assumed to satisfy

$\boldsymbol{y}^{o}\left(t_{n}\right)=\boldsymbol{h}\left[\boldsymbol{x}^{t}\left(t_{n}\right)\right]+\boldsymbol{e}\left(t_{n}\right)$,

where the vector of Gaussian random variable $\boldsymbol{e}\left(t_{n}\right)$ with mean $\mathbf{0}$ and covariance matrix $\mathbf{R}\left(t_{n}\right)$ represents the observation noise. In practice, $\boldsymbol{h}(\boldsymbol{x})$ is an interpolation of the model variables from the model grid points to the locations and times of the observations and a conversion of the model variables to the observed quantities. ${ }^{1}$

In addition to the analysis $\overline{\boldsymbol{x}}^{a}\left(t_{n}\right)$, an ensemble transform scheme also generates an ensemble of analysis perturbations by

$\mathbf{X}^{a}\left(t_{n}\right)=\mathbf{X}^{b}\left(t_{n}\right) \mathbf{W}^{a}\left(t_{n}\right)$.

The analysis perturbations, which are the columns of $\mathbf{X}^{a}\left(t_{n}\right)$, are added to $\overline{\boldsymbol{x}}^{a}\left(t_{n}\right)$ to obtain the members of the analysis ensemble $\boldsymbol{x}^{a(k)}\left(t_{n}\right) ; k=1, \ldots, K$. One approach to compute the weight vector $\boldsymbol{w}^{a}\left(t_{n}\right)$ and the weight matrix $\mathbf{W}^{a}\left(t_{n}\right)$ is through a square-root Kalman filter algorithm (e.g., Tippett et al., 2003).

\subsection{Coupling strategies}

In all four configurations of the coupling considered in this paper, the global background ensemble is obtained by Eq. (4). In the first three strategies we describe, the coupling is in one direction: the limited area data assimilation process uses information provided by the global analysis at the current or the previous analysis time, but the limited area analysis has no effect on the global analysis at the current or future analysis times. In the fourth strategy, the global analysis within the limited area domain is prepared using information from the limited area analysis, thus feeding back information from the limited area data assimilation process to the global data assimilation process. In our description of coupling Strategy 4, we make use of the fact that both the mean analysis and the analysis ensemble members can be computed by linearly combining the background ensemble perturbations.

\footnotetext{
${ }^{1}$ Because the observations assimilated at time $t_{n}$ are collected in the time window $t \in\left[t_{n}-\Delta t / 2, t_{n}+\Delta t / 2\right]$, the model is integrated for a time $\frac{3}{2} \Delta t$ from $t_{n-1}$ to provide a background trajectory $\boldsymbol{x}^{b}(t)$ for the entire observation time window. The observation operator $\boldsymbol{h}$ operates on this background trajectory.
} 


\subsubsection{Strategy 1: limited area analysis by spectral interpolation}

The limited area analysis $\overline{\boldsymbol{x}}_{l}^{a}\left(t_{n}\right)$ is obtained by interpolating the global ensemble mean analysis to the higher resolution grid of the limited area model:

$\overline{\boldsymbol{x}}_{l}^{a}\left(t_{n}\right)=\mathcal{L}\left[\overline{\boldsymbol{x}}_{g}^{a}\left(t_{n}\right)\right]$.

In this configuration, although the global model is run in an ensemble mode, only a single limited area run is prepared using the mean of the global ensemble solution to provide the large scale forcing. In this configuration, the limited area model can outperform the global model if it can develop predictable flow features in response to the higher resolution bottom boundary forcing terms in the limited area domain. (Transient effects, due to the adjustment of the model state to the higher resolution forcing and to the interpolation of the meteorological fields to the higher resolution orography, are expected to have an initial negative influence on the performance of the limited area model.)

\subsubsection{Strategy 2: non-cycled limited area analysis}

Members of the global analysis ensemble at $t_{n-1}$ are interpolated to the higher resolution model grid of the limited area model to obtain a limited area analysis ensemble:

$\boldsymbol{x}_{l}^{a(k)}\left(t_{n-1}\right)=\mathcal{L}\left[\boldsymbol{x}_{g}^{a(k)}\left(t_{n-1}\right)\right], \quad k=1,2, \ldots, K$.

This limited area analysis ensemble is then propagated forward in time using the limited area model to obtain the limited area background ensemble:

$\boldsymbol{x}_{l}^{b(k)}\left(t_{n}\right)=\boldsymbol{f}\left[\boldsymbol{x}_{l}^{a(k)}\left(t_{n-1}\right), \boldsymbol{x}_{g}^{a(k)}\left(t_{n-1}\right)\right]$.

A limited area analysis $\overline{\boldsymbol{x}}_{l}^{a}\left(t_{n}\right)$ is then prepared by applying Eqs. (5-10) the limited area background ensemble $\left\{x_{l}^{b(k)}\left(t_{n}\right)\right.$, $k=1,2, \ldots, K\}$. This procedure is repeated at each analysis time. Note that the limited area analysis obtained at time $t_{n}$ is not used at the subsequent analysis times $t_{m}(m \geq n)$. In this configuration, the limited area analysis can perform better than in Strategy 1, if the high resolution background ensemble perturbations that develop after a $\frac{3}{2} \Delta t$ time integration of the limited area model, in response to the combined effects of uncertainties in the lower resolution large scale flow and the higher resolution forcing terms, provide a more accurate estimate of the background error covariance than does the global background ensemble. Such an approach can also improve the limited area forecasts, if it reduces the transient effects that may occur in Strategy 1 due to using a different model (the global model) in the data assimilation process than in the forecast process (the limited area model). Finally, using a higher resolution model grid in the analysis can reduce the interpolation errors introduced during the computation of the observation operator.

\subsubsection{Strategy 3: cycled limited area analysis}

Strategy 2 is employed to create the limited area analysis ensemble only at the very first analysis time, $t_{1}$. In all subsequent cycles, the limited area background ensemble $\left\{x_{l}^{b(k)}\left(t_{n}\right), k=1,2, \ldots, K\right\}$ is obtained by integrating the limited area model from the limited area analysis ensemble $\left\{\boldsymbol{x}_{l}^{a(k)}\left(t_{n-1}\right), k=1,2, \ldots, K\right\}$ from the previous analysis time, and the limited area analysis ensemble at time $t_{n}$ is obtained by Kalman filter assimilation of observations, as described in Sects. 2 and 2.3. In this configuration, the limited area analysis can perform better than in Strategy 2, if the limited area background uncertainties cannot be fully modeled as a rapid response of the smaller scale uncertainties to the global scale analysis uncertainties. In this case, cycling the limited area analysis may result in a more accurate estimation of the state through a more accurate estimation of the background mean and the background error covariance matrix of the limited area system.

\subsubsection{Strategy 4: feedback from the limited area analysis to the global analysis}

In the three coupling strategies described so far, the global analysis is prepared prior to the limited area analysis both outside and within the limited area domain. In contrast, in the last strategy we describe, the limited area analysis is prepared first in the limited area domain and the weights $\boldsymbol{w}_{l}^{a}\left(t_{n}\right)$ and $\mathbf{W}_{l}^{a}\left(t_{n}\right)$ from the limited area analysis are applied to global background ensemble to obtain the global analysis inside the limited area domain. Outside the limited area domain, the global analysis ensemble is obtained as before, computing the weights based on the global background ensemble.

This strategy introduces a feedback from the limited area analysis process to the global process. An attractive aspect of this approach is that it produces a global analysis ensemble that is consistent with the limited area analysis ensemble, in the sense, that the $k$-th member of the global analysis ensemble, $\boldsymbol{x}_{g}^{a(k)}\left(t_{n}\right)$, and the $k$-th member of the limited area analysis ensemble, $x_{l}^{a(k)}\left(t_{n}\right)$, are obtained by the same linear combination of the background ensemble members. This is appealing because each limited area background ensemble member is dynamically coupled to the global background ensemble member of the same ensemble index (e.g., $\boldsymbol{x}_{l}^{b(k)}\left(t_{n}\right)$ is coupled to $\boldsymbol{x}_{g}^{b(k)}\left(t_{n}\right)$ ); applying the same weights to the limited area and global ensemble members, we ensure that the global analyses $\boldsymbol{x}_{g}^{a(k)}\left(t_{n}\right)$ and the analyses of the high resolution perturbations $\boldsymbol{x}_{l}^{\prime(k)}\left(t_{n}\right)$ are consistent with each other.

The feedback may also improve the global analysis in the area near and within the limited area domain. In particular, using the high-resolution model fields to obtain the global analysis may reduce the effect of the representativeness errors in the observations. There are two practical issues that have to be addressed when implementing Strategy 4. First, 
using the weights from the higher resolution limited area analysis in the global analysis requires an algorithm to map the weights from the high resolution grid to the lower resolution global grid. Second, abrupt changes may occur in the weights near the boundaries of the limited area domain. This can be addressed by implementing a blending process that smooths the changes in the weights near the boundary of the limited area domain (see Sect. 3.2).

Finally, we note that it would be particularly interesting to test Strategy 4 on a coupled global limited-area model with two-way nesting; that is, in a system where the highresolution global solution continuously affects the coarse resolution global solution within the limited area domain (e.g., Harris and Durran, 2010). Unfortunately, while two-way nesting is becoming standard for mesoscale models, there is no readily available two-way nested system for the globallimited-area setting.

\section{Experiment design}

First, we briefly introduce the three main components of our coupled analysis-forecast system: the global GFS model, the limited area RSM model and the LETKF data assimilation system. Then, we describe the design of the numerical experiments, the observational data sets we assimilate, and the verification scores we use to evaluate the different coupling strategies.

\subsection{System components}

\subsubsection{The model component of the GFS}

The GFS consists of a model and a data assimilation component, but in this study we use only the model component. The dynamical core of the model is described in Sela (1980). The model has been upgraded numerous times since the nineteen-eighties, mainly to improve the physical parametrization and the computational performance, but the general solution strategy of the dynamical core has remained the same. In particular, the model uses the spectral transform technique to solve the model equations; that is, the nonlinear terms and the terms associated with parametrized physical processes are computed on a grid, while the spatial derivatives are computed in spectral space using spherical harmonics for the representation of the atmospheric fields. For vertical discretization the model uses a sigma coordinate system. We use a version of the model that was used in operations in 2004, abut at a lover horizontal and vertical resolution. We integrate the model using a triangular truncation with a cut-off wave-number of 62 and 28 vertical sigma levels (T62L28). At this spectral resolution the nominal resolution of the model (the grid spacing) is about $150 \mathrm{~km}$ in the mid-latitudes, but, because of the use of a scale dependent diffusion to maintain a realistic kinetic energy spectrum, the small-scale components of the fields are artificially dampened. The effective resolution of the model is about $200 \mathrm{~km}$ in the mid-latitudes.

\subsubsection{RSM}

The RSM predicts the evolution of a high resolution perturbation to the lower resolution global model solution and obtains the high-resolution model forecast by adding the forecast perturbation to the global forecast (Juang, 1992; Juang et al., 1997). For the computation of the sum of the perturbation and the global fields, the spherical harmonics that represent the global fields in spectral space are directly transformed to the grid points of the NCEP RSM. In our experiments, we use the RSM with a 6-h nesting period, that is, we store the global model solution with a 6-h resolution and compute the global fields by a linear time interpolation at each time step of the integration of the RSM.

In the RSM, the time evolution of the perturbation is governed by the nonlinear interactions between the different components of the perturbation and the nonlinear interactions between the perturbations and the large scale flow. The perturbation equation is solved by a spectral transform technique using double cosine and sine functions to represent the meteorological fields. The vertical coordinate of the model is sigma and the 28 sigma levels of the RSM in our experiments are the same as those of the GFS model. We choose an extended North-American region to be the limited area domain (Fig. 1), and we use a horizontal resolution of $48 \mathrm{~km}$ in this domain. While the increase of the model resolution with respect to the resolution of the global model may seem to be modest, locally it can lead to a $300-500 \mathrm{~m}$ more accurate representation of the orography (Fig. 2).

The RSM model offers different options to ensure a smooth transition of the limited area perturbations to zero at the boundaries. We choose the implicit relaxation and blending procedures described in Juang et al. (1997). The blending procedure computes a weighted average of the high resolution forecast and the global forecast, giving increasingly larger weights to the global forecast moving toward the boundaries. We set the blending parameters such that blending affects a total of $20 \%$ of the grid points in each direction (10\% at each lateral boundary). Thus, since the total number of grid points in the zonal direction is 193 , the blending affects 19 points at the eastern and western boundaries and 14 of the 140 grid points in the meridional direction at the southern and northern boundaries. Multiple nesting and nonhydrostatic options are available in the RSM (Juang et al., 1997; Juang, 2000), but we do not use these features in our experiments.

\subsection{LETKF}

In the LETKF, the state update step of the Kalman filter is performed independently for each component of the state 


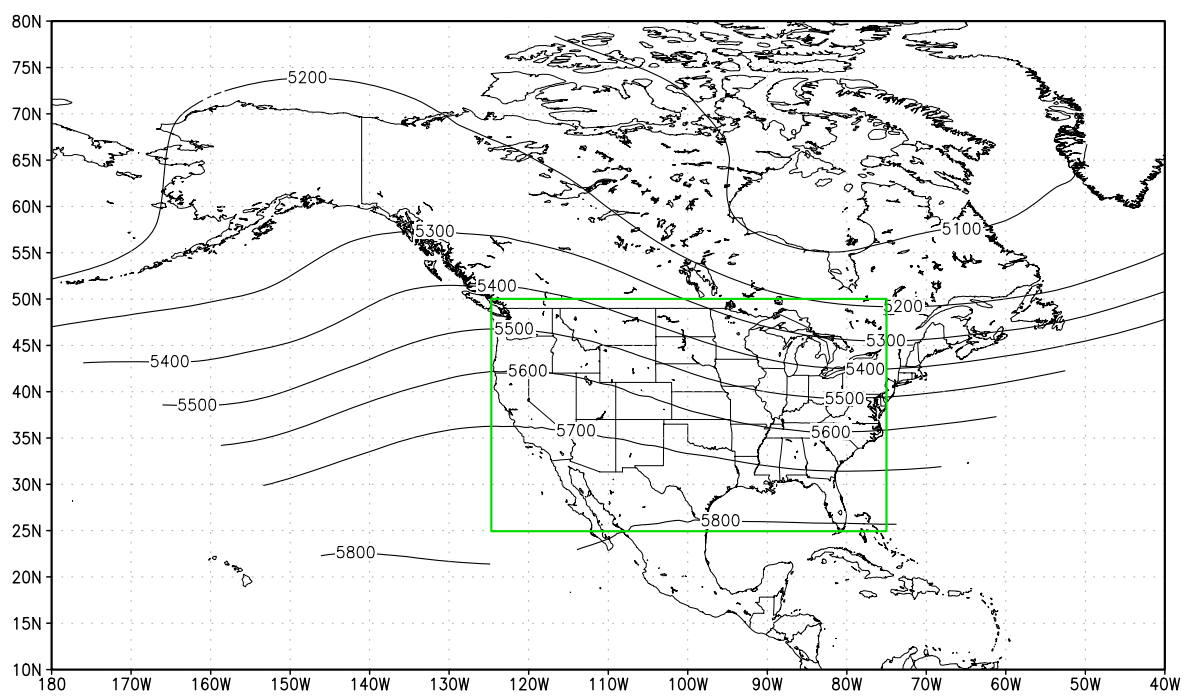

Fig. 1. The limited area domain. Green rectangle indicates the boundary of the forecast verification domain. Thin contours show the time mean of the geopotential height of the pressure level in the "true" states.

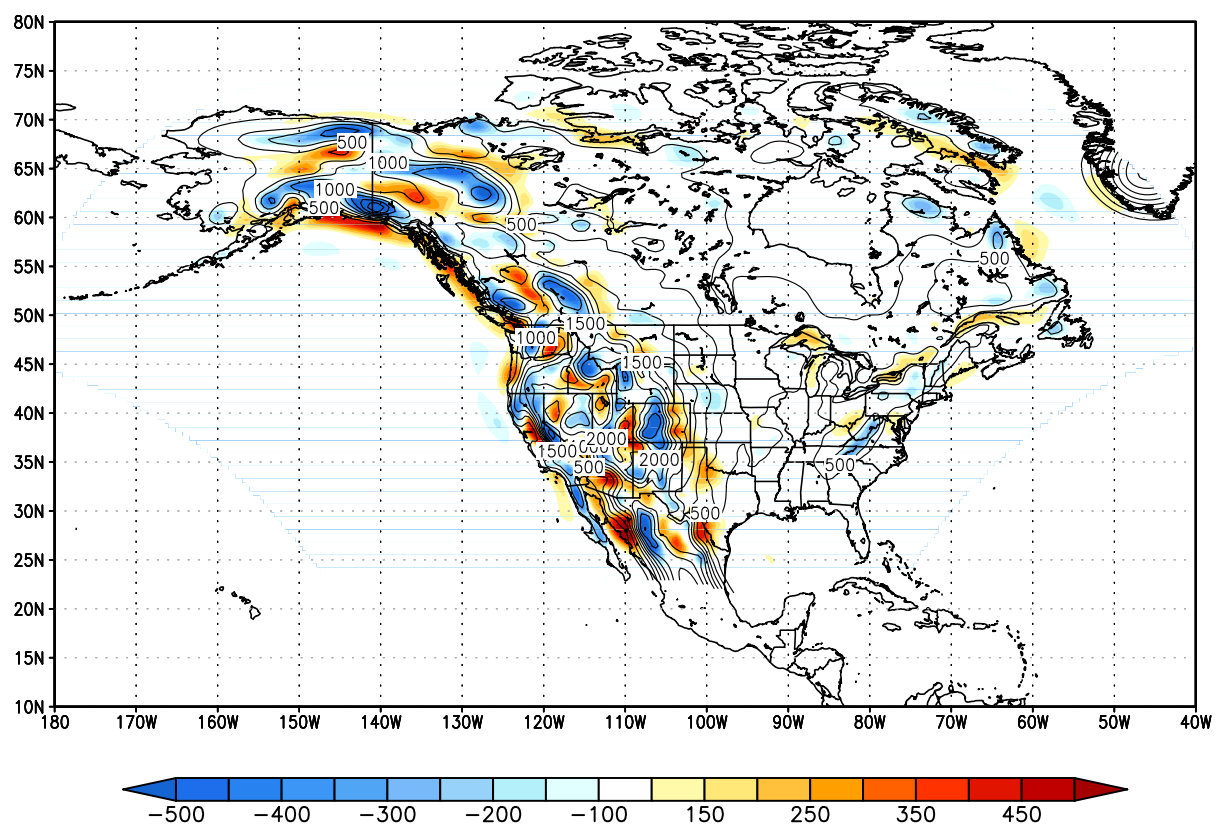

Fig. 2. Difference in the orography of the RSM and the GFS models. Thin contours show the height of the orography in the RSM model, while color shades show the height of the orography in the GFS model minus the height of the orography in the RSM.

vector (Ott et al., 2004; Hunt et al., 2007). A key step of the LETKF algorithm is the selection of the set of observations that are considered when updating the estimate of a given state vector component. In practice, the different state vector components at a given grid point are analyzed in one step and in situ observations are selected for assimilation if they are closer to the grid point than a given distance. The assimilation of nonlocal radiance observations with the LETKF is also possible, but for those observations the observation selection is done in a different way (Fertig et al., 2008). In this study, we use the same set of LETKF parameters in both the global and the limited area data assimilation system as we used in the global system described in Szunyogh et al. (2008). The number of ensemble members is $K=40$, observations are assimilated if they are in a $800 \mathrm{~km}$ radius of the grid point, and the inverse of the assumed observational error variance is tapered linearly from one over its original value to zero between a distance of 500 and $800 \mathrm{~km}$ (thus tapering the effects of observations on the analysis that are further away than $500 \mathrm{~km}$ ). The initial ensemble members are sampled 


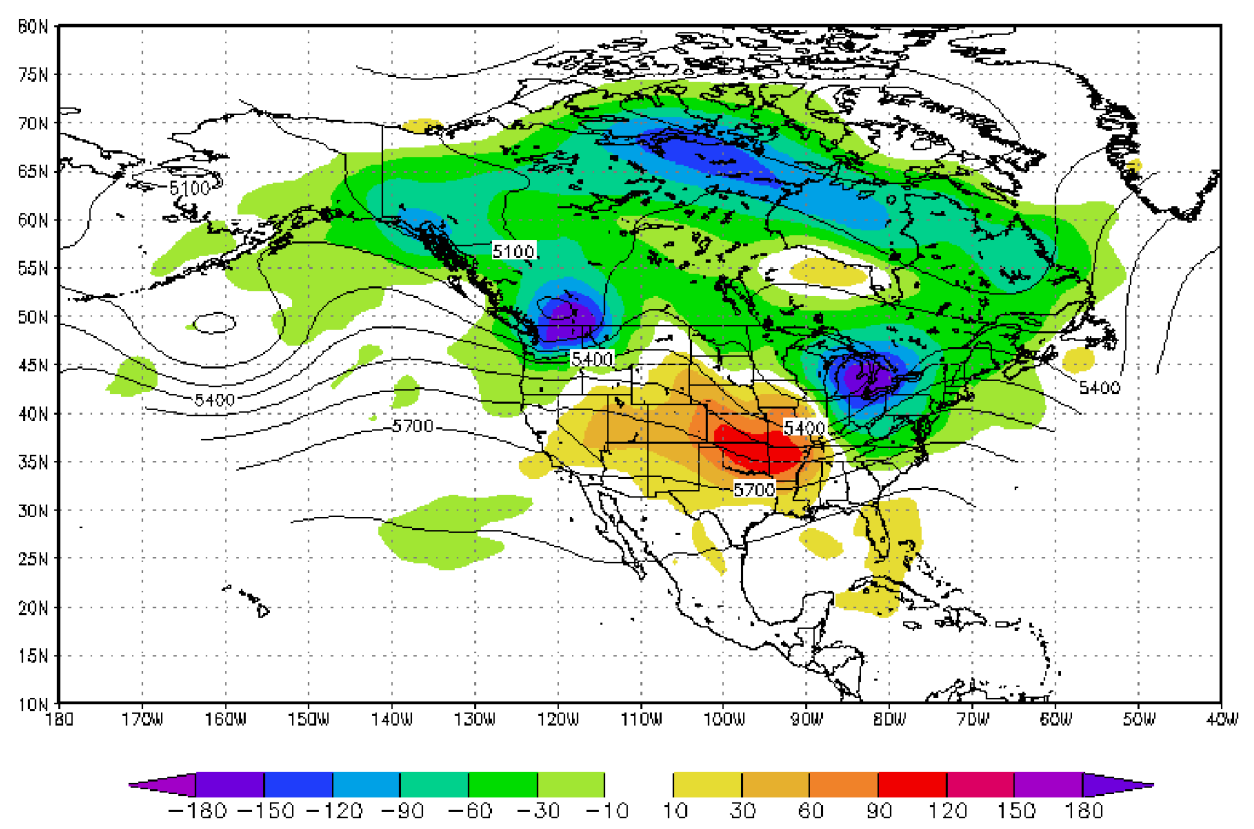

Fig. 3. A snapshot of the high-resolution perturbation component of the truth in the limited area domain (color shades). Shown is the $500 \mathrm{hPa}$ geopotential height component of the perturbation after 16 days of model integration. Also shown is the low resolution global component of the $500 \mathrm{hPa}$ "true" geopotential height field (contours).

from a free run with the NCEP GFS. The one important difference between our implementation of the LETKF on the GFS and the RSM is that in the GFS implementation we employ a digital filter (Lynch and Huang, 1992) to control free gravity wave oscillations, but we do not employ such filtering for the RSM.

In our implementation of Strategy 4, we compute the weights $\boldsymbol{w}_{g}^{a}\left(t_{n}\right)$ and $\mathbf{W}_{g}^{a}\left(t_{n}\right)$ for the global analysis within the limited area domain by taking the algebraic mean of the weights at the four closest grid point of the high resolution grid. We found that the blending procedure applied by the RSM to the model fields results in a sufficiently smooth transition of the weights of the global system near the boundaries. Thus, applying a blending algorithm directly to the weights was deemed not necessary.

\subsection{Observational data set}

The observational data set is identical to the one used in Szunyogh et al. (2008). It includes all conventional (non-radiance) measurements that were operationally assimilated at NCEP between 1 January 2004 at 00:00 UTC and 29 February 2004 18:00 UTC. In the simulated observation experiments, we use information only about the location and type of the observations from the observation reports to generate simulated observation of the right type at realistic locations by adding random "observation noise" to a time series of "true" states. To simplify the generation of the simulated observations, we assume that all observations were taken at the analysis time; that is, the random observational noise is added to the true state at the analysis time. ${ }^{2}$ This true state is obtained by first generating a time series of coarse resolution global true states with a 60-day integration of the NCEP GFS starting from the operational global analysis of NCEP on 1 January 2004 at 00:00 UTC. Then, a 60-day integration of the RSM is carried out to add a high resolution perturbation to the true state over the extended North American region. Figure 1 shows the time mean flow in the nature run. One important feature of the time mean flow is the low pressure region over north-east Canada. The limited area model domain includes only part of this stationary low, and, as we show later, this will obviously degrades the performance of the limited area system in the region north of the $5200 \mathrm{gpm}$ isoline.

Figure 3 shows the difference between the high- and the low-resolution components of the true state for the $500 \mathrm{hPa}$ geopotential height after 16 days of integration. Although the regional model is started from a global analysis and the global component of the high-resolution fields is provided by the NCEP GFS throughout the entire simulation, substantial differences develop between the high resolution true state and its global component inside the limited area domain. For a more quantitative assessment of the difference between the

\footnotetext{
${ }^{2}$ When observations of the real atmosphere are assimilated, we take advantage of the 4-dimensional capabilities of the LETKF (Hunt et al., 2004, 2007); that is, we use background information, regarding both the state and the error covariance matrix, which is valid at the exact observation time.
} 


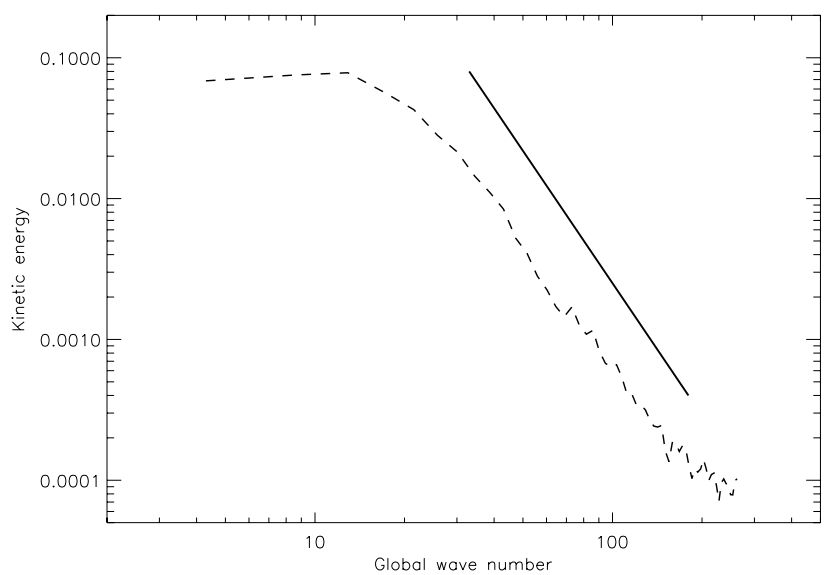

Fig. 4. The time mean kinetic energy spectrum of the highresolution perturbation component of the truth with respect to the global wave number (dashes) in a log-log scale. The straight solid line with slope -3 indicates the scaling law for the kinetic energy in the inertial range of two-dimensional turbulence.

high-resolution nature run and its low-resolution component, we show the kinetic energy spectrum for the $\boldsymbol{x}_{l}^{\prime}(t)$ perturbation component of the nature run (Fig. 4). The spectral analysis takes advantage of the property of $\boldsymbol{x}_{l}^{\prime}(t)$ that it satisfies double-periodic boundary conditions on the limited area domain: the kinetic energy spectrum is obtained by (i) taking the double Fourier transform of the two zonal components of the wind vector, (ii) computing the square of the magnitude of the spectral coefficients by multiplying the spectral coefficients with their complex conjugate, (iii) adding the square of the magnitude for the two components of the wind (iv) summing the squares in unit width wave number bands centering the bands on the integer wave numbers, finally (v) taking the time mean of the spectra. To make the interpretation of the figure easier, we scale the wave numbers by the ratio of the length of the full latitude circle along the globe at the given latitude and the zonal length of the limited area domain. That is, the results from the limited area spectral analysis are shown with respect to the global wave number. The spectral analysis shows that the initially zero perturbation $\boldsymbol{x}_{l}^{\prime}(t)$ develops an energy spectrum that peaks at the synoptic scales (wave number 6-20) and has a relatively large value even at the largest resolved scale of about wave number 4 .

\subsection{Verification scores}

We assess the performance of the different coupling strategies by preparing deterministic global and limited area forecasts for each configuration of the global/local data assimilation coupling. We verify forecasts at $12-\mathrm{h}$ and the 48 $\mathrm{h}$ forecast lead times. Since the boundary conditions tend to degrade the limited area forecasts near the boundaries (e.g., Warner et al., 1997; Torn et al., 2006), we define a
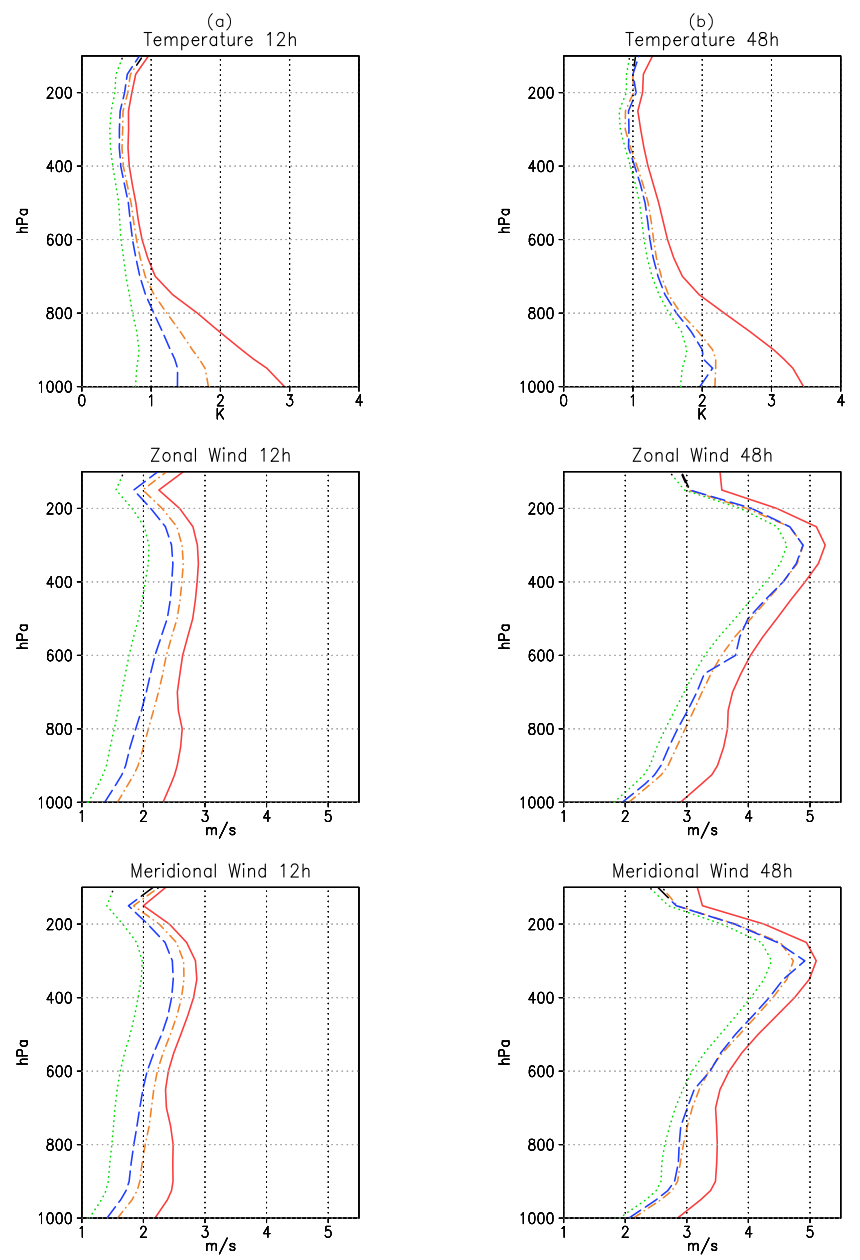

Fig. 5. Vertical profile of the root-mean-square forecast error in the limited area domain at 12-h (a) and 48-h (b) forecast time for the global forecast (red solid) and for the limited area forecasts with coupling Strategies 1 (orange dashes and dots), 2 (blue dashes) and 3 (green dots).

verification region that is smaller than the limited area domain. (See Fig. 1 for the definition of the verification region.) We deem a configuration better if it produces, on average, more accurate forecasts in the verification domain.

In the perfect model experiments, the verification is done against the known true state on the high resolution grid of the RSM. This verification approach requires the interpolation of the global model fields onto the higher resolution limited area grid, when verifying global fields. The magnitude of the error of the forecast of a variable at a given time and model level is measured by the root-mean-square error. The mean in the computation of the root-mean-square is taken either over all grid points in the verification domain and over all verification times, or over all verification times. In the former case, the error for a given variable and level is a single scalar, while in the latter case, the error is a two-dimensional field in the limited area domain. 
(a) 12-Hour Forecast

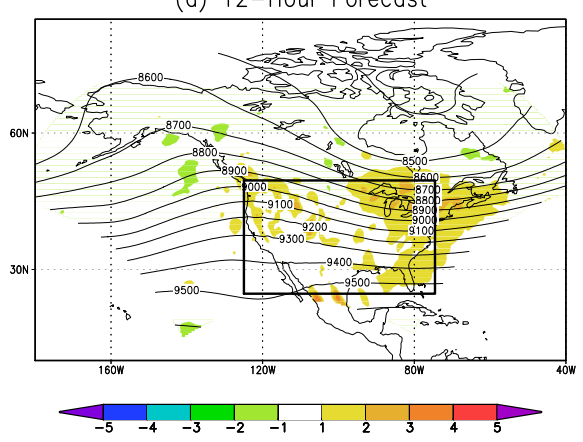

(b) 12-Hour Forecast

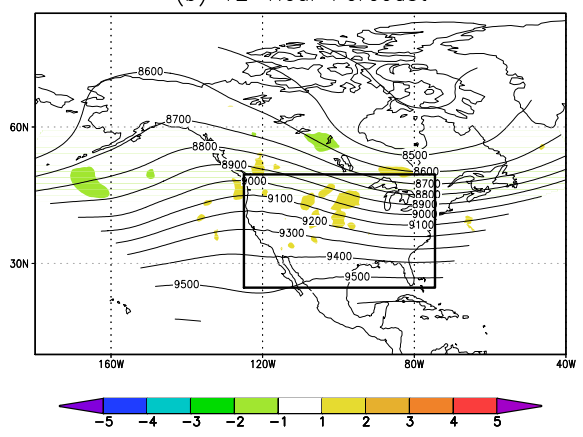

(c) 12-Hour Forecast

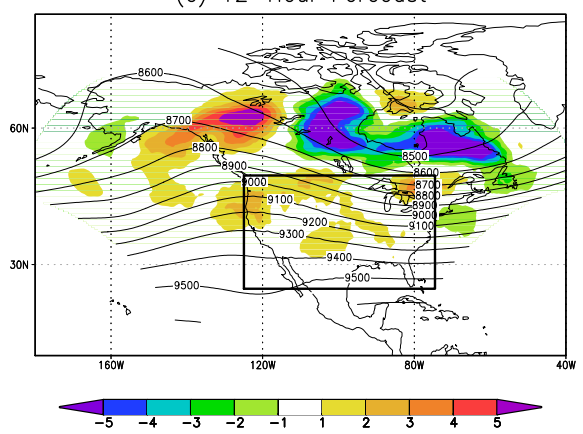

(d) 48-Hour Forecast

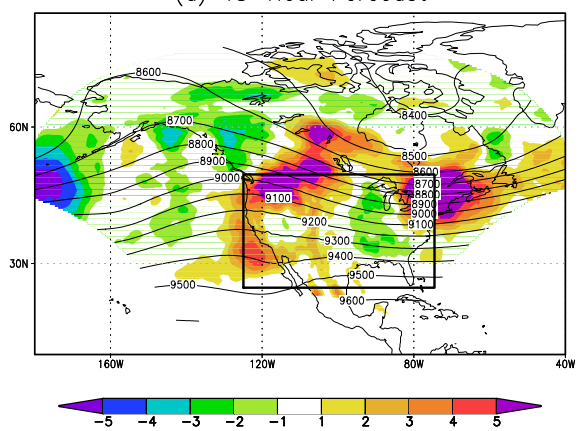

(e) 48-Hour Forecast

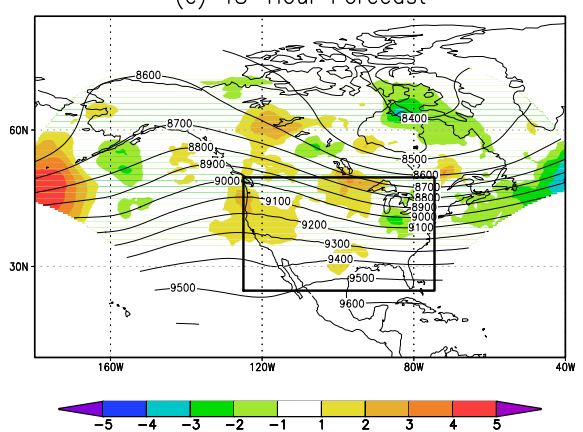

(f) 48-Hour Forecast

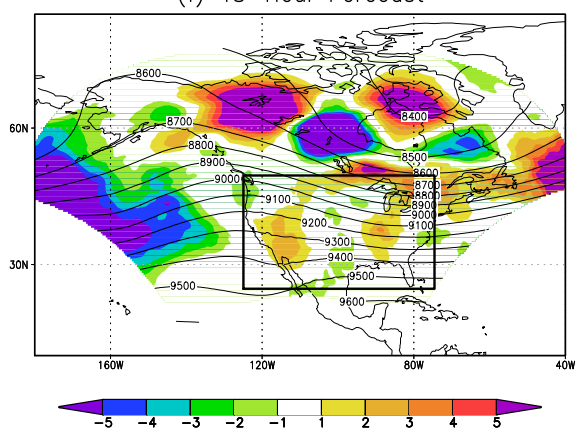

Fig. 6. The difference between the root-mean square errors of the geopotential height forecasts at the $300 \mathrm{hPa}$ level for the different configurations of the analysis system at 12-h and 48-h lead times. Shown are the difference between the forecasts started from the global analysis and the limited area analysis of Strategy 1 (a and $\mathbf{d})$, from the limited area analyses of Strategies 1 and 2 (b and e), from the limited area analyses of Strategies 2 and 3 (c and $\mathbf{f}$ ). Where the values are positive the forecast from latter analysis is more accurate. Also shown is the mean flow at the $300 \mathrm{hPa}$ level for the "true states" in the verification period (contours).

To verify the forecasts of the real atmosphere, we compute the root-mean-square error of the forecasts against radiosonde observations at the mandatory pressure levels where observations are available for all radiosondes. For the computation of this statistics, the forecasts are interpolated by a bilinear horizontal interpolation to the observation locations and the mean is taken over all observation locations and over all observation times. Since the errors in the forecasts are not correlated with the errors in the radiosonde observations, this verification approach can reliably detect changes in the accuracy of the forecasts that are smaller than the root-mean-square of the observation errors (e.g., Szunyogh et al., 2000).

\section{Results for the perfect model scenario}

We first compare the performance of data assimilation systems based on Strategies 1-3. Then we compare the performance of the two systems based on Strategy 3 and 4. 
(a) 12-Hour Forecast

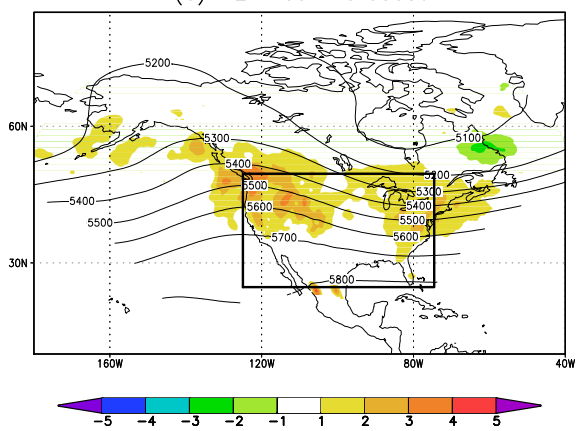

(b) 12-Hour Forecast

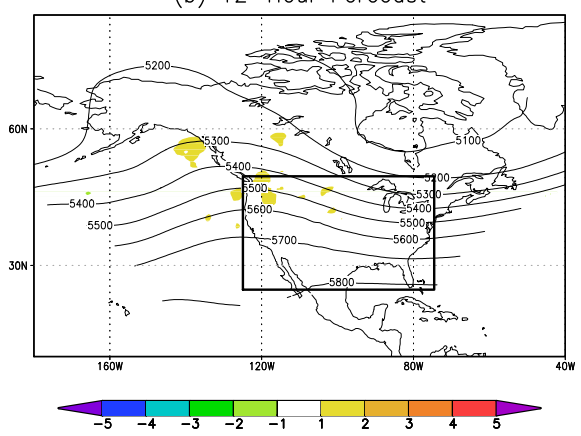

(c) 12-Hour Forecast

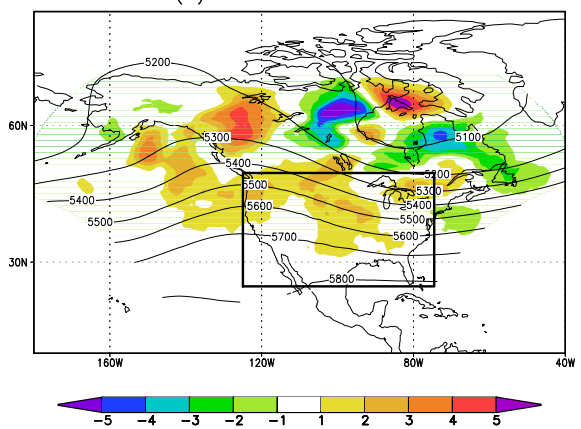

(d) 48-Hour Forecast

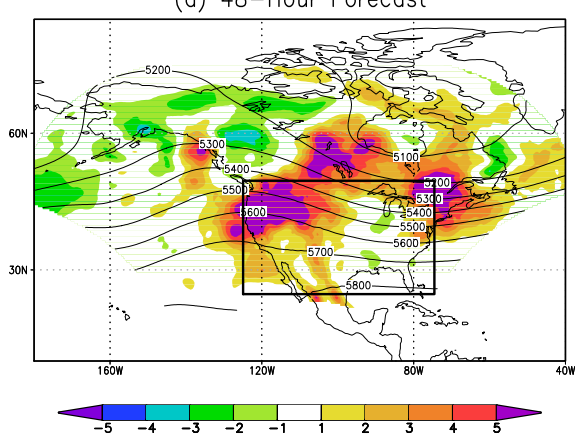

(e) 48-Hour Forecast

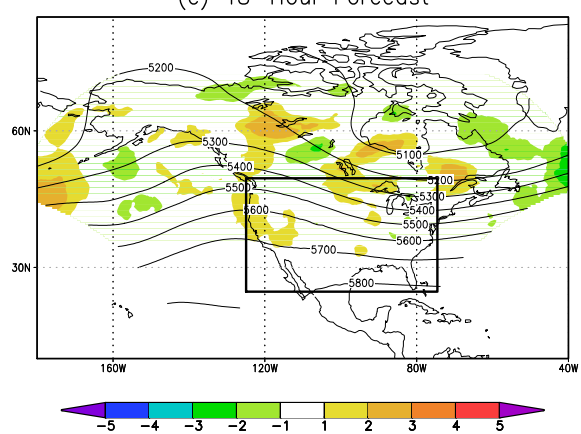

(f) 48-Hour Forecast

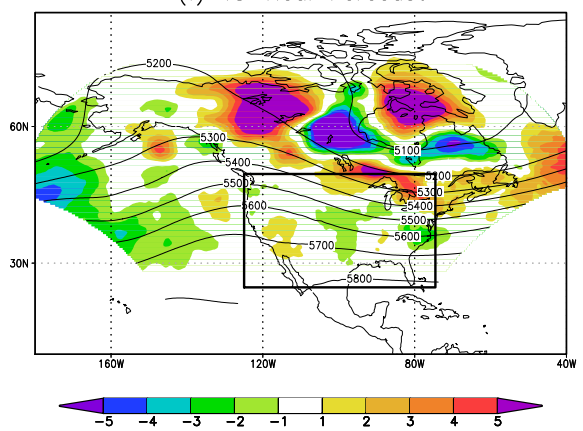

Fig. 7. Same as Fig. 6, except for the geopotential height forecast at the $500 \mathrm{hPa}$ level.

\subsection{The comparison of Strategies 1, 2 and 3}

In Fig. 5, we show the vertical profile of the root-meansquare error at 12 -h and 48 -h forecast times for the temperature and the two horizontal components of the wind. The results suggest that all three limited area strategies provide forecasts, which are more accurate than the global forecast. On average, Strategy 3 provides more accurate forecasts than Strategy 2, and Strategy 2 provides more accurate forecasts than Strategy 1. While all three limited area forecast systems maintain their large advantage over the global system for the entire 48-h, the difference between the performance of the three limited area systems is smaller at 48-h than at 12-h forecast time.
We show the spatial distribution of the forecast improvements introduced by the increasingly more sophisticated limited area data assimilation process for the geopotential height at the 300 and $500 \mathrm{hPa}$ (Figs. 6 and 7) and for the temperature at $850 \mathrm{hPa}$ pressure level (Fig. 8). Using the limited area model only to prepare the forecasts (Strategy 1) consistently improves all verified forecast parameters in the verification domain (see panels a and d of Figs. 6-8), evolving the backround ensemble for a single assimilation cycle with the limited area model (Strategy 2) has little additional value compared to Strategy 1 (panels b and e); while cycling the limited area assimilation (Strategy 3) leads to major improvements compared to Strategy 2. While the system based on Strategy 3 performs the best in the verification domain, 
(a) 12-Hour Forecast

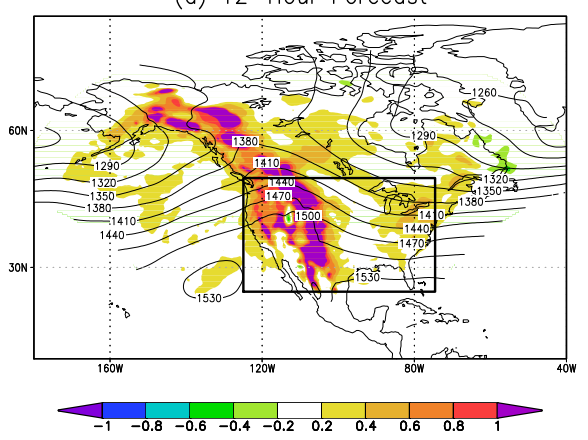

(b) 12-Hour Forecast

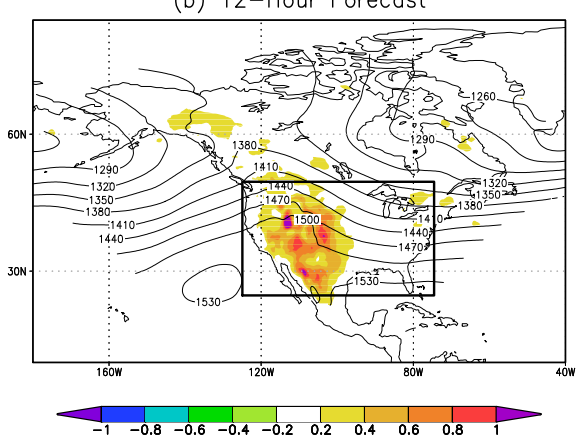

(c) 12-Hour Forecast

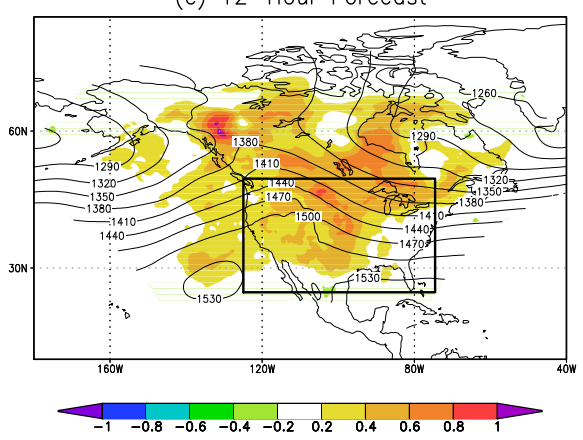

(d) 48-Hour Forecast

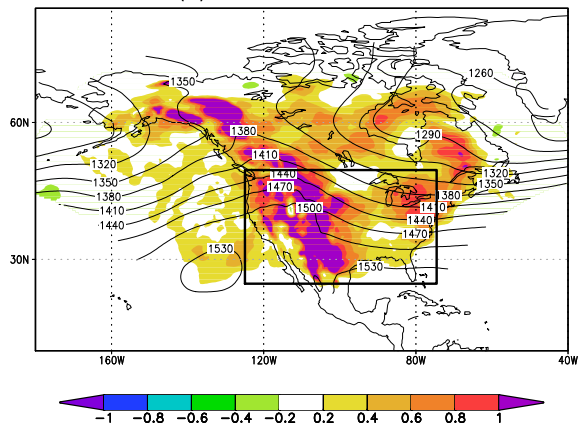

(e) 48-Hour Forecast

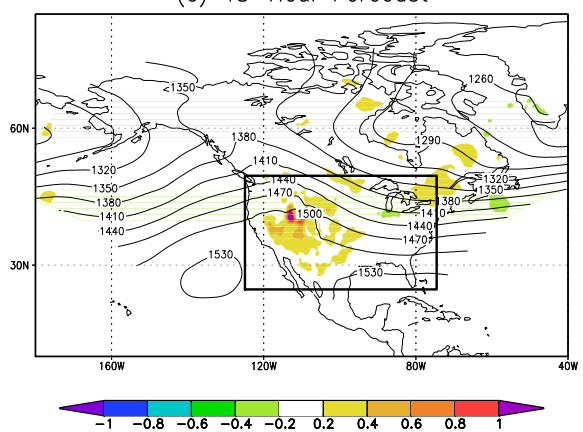

(f) 48-Hour Forecast

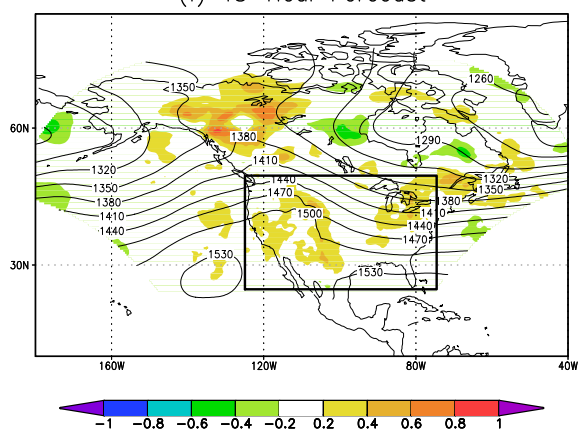

Fig. 8. Same as Fig. 6, except for the temperature at the $850 \mathrm{hPa}$ level.

that system also has obvious difficulties outside the verification domain, near the northern and western boundaries of the limited area domain. This result suggests that while propagating the background covariance information with the limited area model is beneficial in general, propagating the covariance through the boundaries can be problematic even in the perfect model scenario.

\subsection{The comparison of Strategies 3 and 4}

Area averaged errors are not shown for Strategy 4, because we do not find statistically significant differences between Strategies 3 and 4 for these statistics. (We tested the statistical significance of the difference between the errors for the two strategies by the procedure described in Szunyogh et al., 2008.) We show results, however, for the 48-h forecast lead time (Fig. 9): Strategy 4 improves the limited area forecasts within the verification region and outside the verification region near the western boundary of the limited area domain, but it degrades the forecasts north of the verification region (panel a, b, and c). This result indicates, that while Strategy 4 has the potential, in general, to improve the limited area forecasts, in regions where the limited area domain captures only a part of an important large scale forecast feature, feeding back information from the limited area system can have negative effects. The right hand side panels of Fig. 9 (panels d, e, and f), which show the effect of the feedback on the global analysis in the limited area domain, indicate a dominantly negative effect on the global forecasts of the feedback on the global forecast. 
(a)

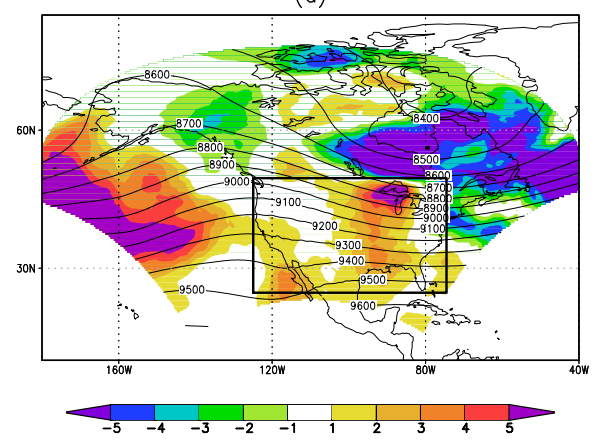

(b)

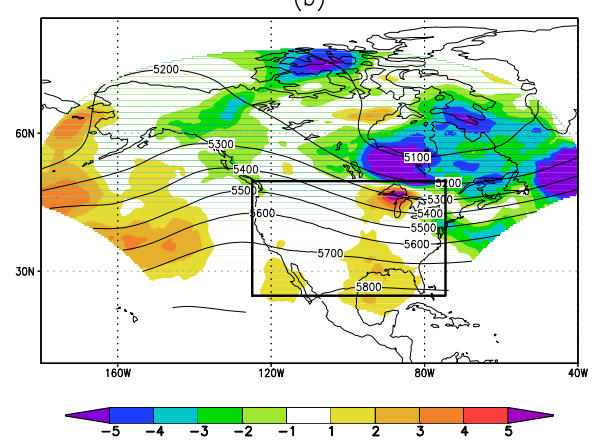

(c)

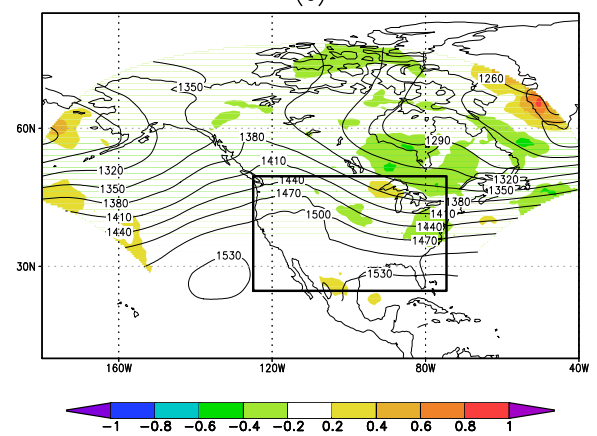

(d)

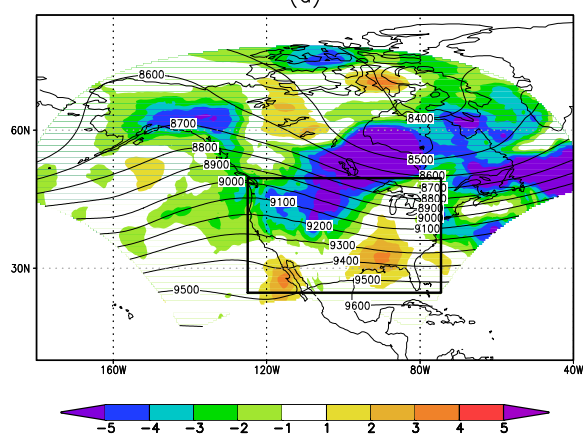

(e)

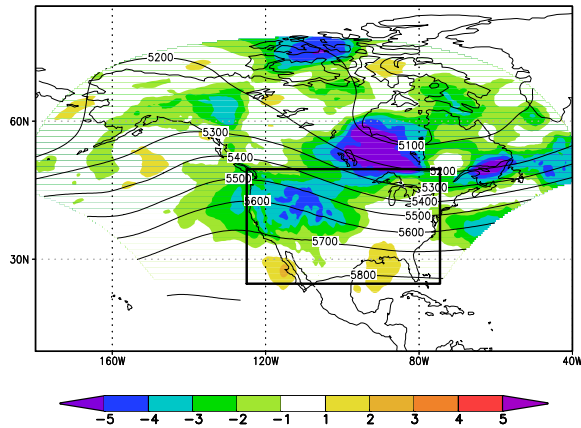

(f)

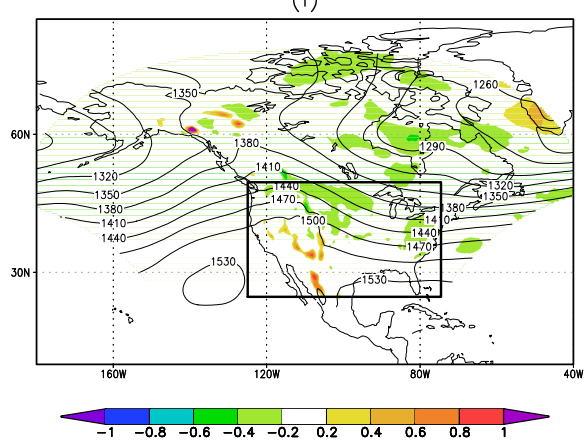

Fig. 9. The difference between the root-mean square errors of the 48-h forecasts started from the analyses obtained by Strategy 4 and Strategy 3. Results are shown for the limited area geopotential height forecasts at the $300 \mathrm{hPa}$ (a) and the $500 \mathrm{hPa}(\mathbf{b})$, and the limited area temperature forecasts at $850 \mathrm{hPa}$ (panel $c$ ); for the global geopotential height forecasts at $300 \mathrm{hPa}(\mathbf{d})$ and $500 \mathrm{hPa}(\mathbf{e})$ and the global temperature forecast at $850 \mathrm{hPa}$ (f). Strategy 4 provides more accurate forecasts where the shades indicate positive values. Contour show the time mean of the true geopotential height at the given level.

\subsection{Spectral analysis}

Figure 10 shows the spectral distribution of the error in the zonal wind forecasts for strategies 1,2 and 3 at both the 12-h and the 48-h forecast times. This figure is produced the same way as Fig. 4, except that the Fourier transform is applied to the errors in the high-resolution wind forecast perturbation. Since global component of the forecast is the same for all three strategies, this figure illustrates the difference between the spectral distribution of the error in the limited area perturbation component of the forecast for the three strategies. Results are not shown for Strategy 4, because in that case the difference between errors in the large scale forecasts also contributes to the difference between the errors for Strategy 4 and not for the other strategies.

The most striking feature of Fig. 10 is the large advantage of the system that cycles the limited area analysis (Strategy 3) at 12 -h forecast time in the wave number range $10-40$. This result indicates that the LETKF coupled with the RSM can more skillfully predict the covariance in the wave number 

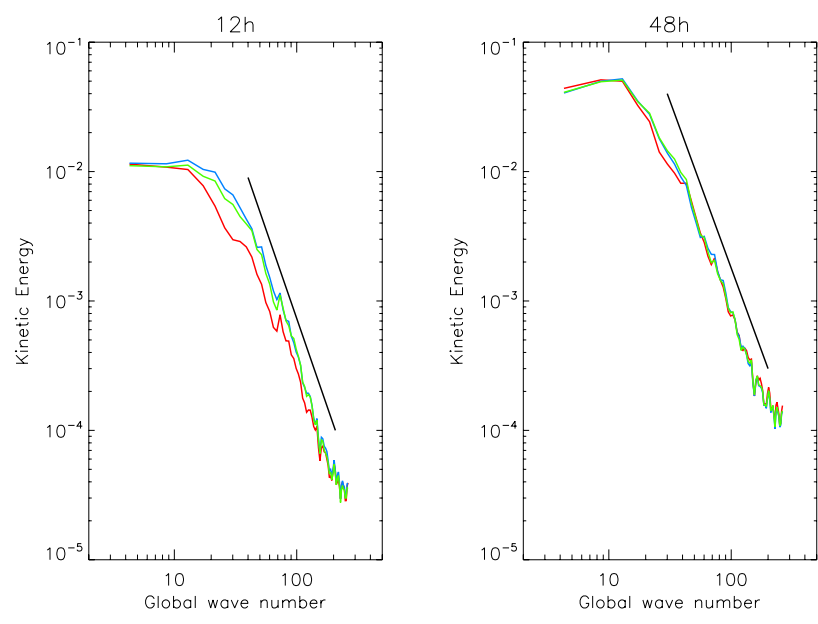

Fig. 10. The kinetic energy spectrum of the forecast error with respect to the global wave number at 12 -h and 48 -h forecast lead times in a log-log scale. Shown is the error for Strategy 1 (blue), Strategy 2 (green) and Strategy 3 (red). The straight solid line with slope -3 indicates the scaling law for the kinetic energy in the inertial range for two-dimensional turbulence.

range $10-40$ when the analysis is cycled. At 12 -h forecast time, the difference between the performance of the different configurations of the system is small at the longest resolved scales (wave numbers larger than 10) and at the shortest resolved scales (wave numbers larger than 60). There is no real difference at 48 -h between the performance of the three configurations, with the exception of a slight advantage of the cycled system (Strategy 3).

\section{Results with observations of the real atmosphere}

\subsection{Comparison of Strategies 1, 2, and 3}

Verification results for our analysis-forecast experiments using observations of the real atmosphere are shown in Fig. 11. Overall, the limited area systems perform slightly better than the global system at 12-h forecast time, while the global system performs better than the limited area systems at 48-h forecast time. The difference between the performance of the limited area systems and the global system is larger for the two components of the wind than for the temperature. In particular, the clear advantage of the limited area systems for the zonal component of the wind below the $300 \mathrm{hPa}$ level at 12-h lead time turns into a clear disadvantage by the 48 -h forecast time. Another interesting feature of the verification results for the two components of the wind is the big advantage of the global system in the upper troposphere (above $300 \mathrm{hPa}$ ), most of which disappears by the 48 -h forecast time. One possible explanation is that the poorer performance of the limited area systems at 12 -h forecast time may be due to vertically propagating spurious gravity waves. Such waves may (a)
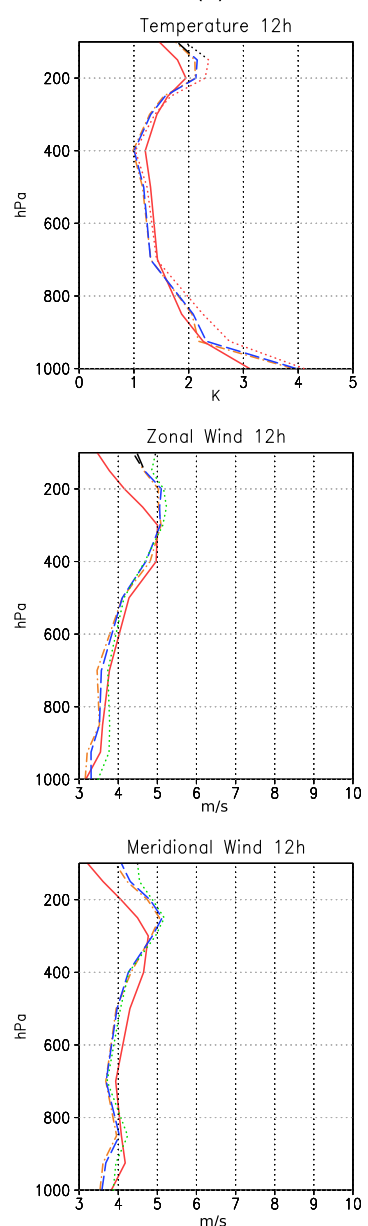

(b)
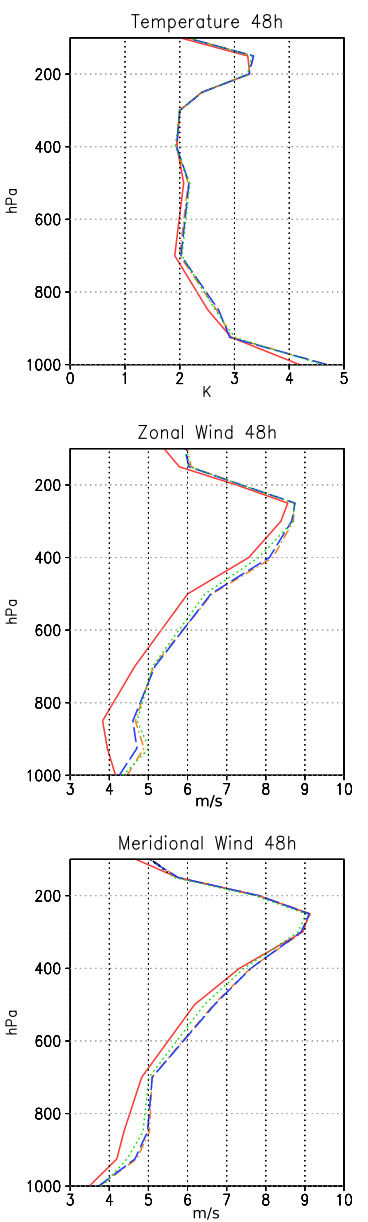

Fig. 11. Vertical profile of the root-mean-square forecast error in the limited area domain at 12-h (a) and 48-h (b) forecast time for the global forecast (red solid) and for the limited area forecasts with coupling strategies 1 (orange dashes and dots), 2 (blue dashes) and 3 (green dots) assimilating observations of the real atmosphere.

play a more important role in the limited area model than in the global model either because of the the lack of initialization or because of a less careful tuning of the mountain drag parametrization for the higher resolution orography of the RSM. A further investigation of this issue is beyond the scope of the present paper.

Similar to the results for the perfect model scenario, there is not much difference between the systems based on the different coupling strategies at 48 -h lead time. However, the picture is very different from what we observed for the perfect model scenario at 12-h forecast time: Strategy 3, which performed the best under the perfect model scenario, performs the worst in the realistic case, while Strategy 2 maintains its slight advantage over Strategy 1. This suggest that the RSM at the tested resolution is not a sufficiently better model than the GFS in the limited area to compensate for the problems that arise at the boundaries in Strategy 3. 
(a)
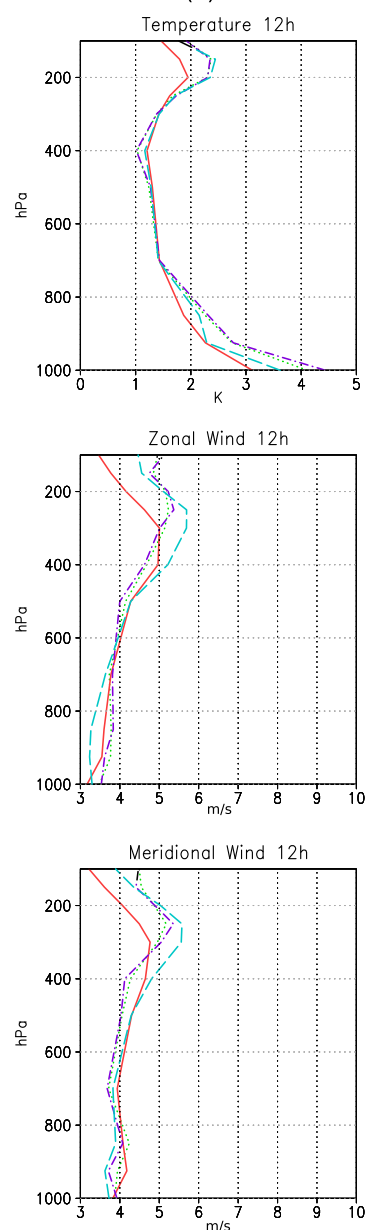

(b)
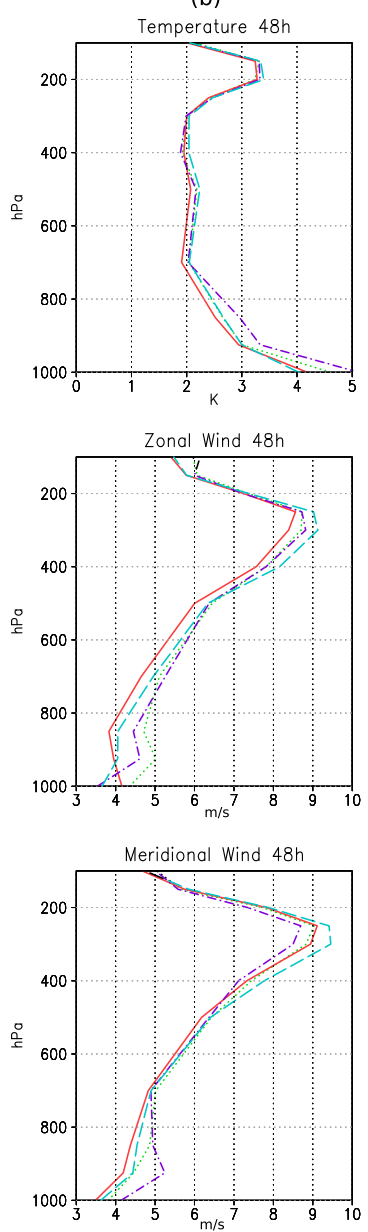

Fig. 12. Results for experiments assimilating observations of the real atmosphere. Vertical profile of the root-mean-square forecast error in the limited area domain at 12-h (a) and 48-h (b) forecast time for the global forecast (red solid), the global forecast with Strategy 4 (cyan dashes), the limited area forecast for Strategy 3 (green dots), and the limited area forecast Strategy 4 (purple dashes and dots).

\subsection{Comparison of the Strategy 3 and 4}

The comparison of the performance of strategies 3 and 4 with real observations is shown in Fig. 12. In these figures, we show the impact of the feedback on both the limited area and global forecasts (the two curves without feedback are the same as in Fig. 12). We note that some caution should be exercised when interpreting the results shown in this pair of figures: the difference between the errors shown in these figures are statistically not significant when tested using the approach of Szunyogh et al. (2008). That test compares the time (sample) mean of the instantaneous differences between the root-mean-square-errors for variance of the two configurations at the different verification times to the variance of the same differences. The failure of the test indicates that the

differences in the errors are not due to consistent differences at the different verification times. Instead, they are the net result of differences that are highly variable in magnitude and sign.

Interestingly, at 12 -h forecast time, the feedback has a much larger effect on the performance of the global forecast than on the performance of the limited area forecast. In particular, while the global forecasts of the temperature is clearly degraded by the feedback above $300 \mathrm{hPa}$ and below $700 \mathrm{hPa}$, and the two horizontal wind components above $500 \mathrm{hPa}$ are clearly degraded, the feedback improves the global forecasts of the two wind components in the lower troposphere (below $500 \mathrm{hPa}$ ). At 48-h lead time, the best of the four forecasts is clearly the global forecast without feedback. The only parameters for which this system is not the best is for the meridional component of the wind in the jet layer (around $300 \mathrm{hPa}$ ), where the limited area system with feedback is the best; and for the zonal component of the wind at the bottom of the atmosphere, where the two systems with feedback are the best. Also, at 48-h lead time the differences are larger between the performance of the two limited area systems. In particular, the system with feedback performs better for the meridional wind component in the jet layer and for the zonal wind below $800 \mathrm{hPa}$, and it performs worse for the temperature and the zonal component of the wind at the bottom of the atmosphere.

\section{Conclusions}

This paper documents our first attempt at exploring the potential benefits of coupling the global and limited area ensemble Kalman Filter data assimilations. To the best of our knowledge, ours is the first study that considers a feedback from the limited area data assimilation process to the global process. We carried out analysis-forecast experiments under a perfect model scenario, where the limited area model was considered to be perfect in the limited area domain and the global model errors is considered to be perfect elsewhere. Since the perfect model experiments have limited value when designing practical data assimilation systems, we also carried out experiments in a more realistic setting, assimilating observations of the real atmosphere. Due to the low spatial resolution of our models and the lack of assimilation of the satellite data, our conclusions are not applicable directly to the operational systems. Instead, they should be viewed as motivation for continued research on the coupling of global and limited area data assimilation.

Our most important findings for the perfect model scenario are the following:

- In the limited area domain, the limited area systems based on the different coupling strategies perform better than the global system. The advantage of the limited area systems is much larger at 12 -h lead time than at 48-h lead time. 
- Preparing a limited area analysis with a cycled limited area system enhances the performance of the limited area forecast system. The main benefit of cycling the limited area analysis is it provides better 12-h lead time forecasts at the synoptic and sub-synoptic scales (global wave number 10-40). A single analysis cycle does not provide sufficient time to achieve a similar effect.

Our additional findings from our tests using real atmospheric data are the following:

- The results with observations of the real atmosphere confirmed that the limited-area data assimilation has potentially larger benefits at the shorter forecast times (12$h$ vs. 48 -h in our experiments). The advantage of the limited area systems is smaller than in the perfect model scenario at $12-\mathrm{h}$ forecast time and has a disadvantage at 48-h forecast time.

- Our attempt to feed back information from the limited area analysis to the global analysis led to mixed results. The feedback improved the 48-h high resolution wind forecast under the perfect model scenario and the meridional large scale wind forecast at 48 -h in the realistic scenario, but also led to considerable degradation of some of the other verified atmospheric variables.

We emphasize that we consider the current study only to be the first step toward exploring the benefits of coupling the global and limited area data assimilation process. One potential extension of the present study would be to focus on the verification of weather parameters (e.g., precipitation) near the surface, where the higher resolution representation of surface effects (e.g., those related to orography) may increase the relative value of the limited area system. Another potential extension would be to increase the ratio of the resolution of the two models from the current 1:4 ratio $(48 \mathrm{~km}$ vs. about $200 \mathrm{~km}$ ). Since, in the current system the cut-off wave number for both models is within the inertial range of two-dimensional turbulence, the regional model does not really bring in new physics compared to the global model. Increasing the resolution of the limited area model to a range where some of the non-hydrostatic processes are explicitly resolved would bring in a new source of kinetic energy (convection), as well as the effects of three-dimensional turbulence. Bringing in new physics could reduce the representativeness component of the observation errors with respect to the limited area model dynamics. This, in turn, could be expected to increase the potential benefits of feeding back information from the limited area data assimilation system to the global data assimilation system. One particular area of research where we expect such an approach to be especially beneficial is in the verification of interaction between a tropical cyclone and the large scale flow. We are currently in the process of testing our coupled data assimilation system for such a scenario.
Strategy 4 is one particular way to interpolate the high resolution analysis onto the grid of the lower resolution global model. This approach takes advantage of the close relationship between the high resolution limited area model fields and the low resolution global fields in the NCEP RSM. We are currently in the process of developing a more general coupling strategy that would work well when the limited area model and the global model are less closely related. We will report on this effort in a future paper.

Acknowledgements. The authors thank Briant Hunt, Eric Kostelich and Gyorgyi Gyarmati for their contribution to this work. The research reported in this paper was funded by the National Science Foundation (Grants ATM-0722721 and 0935538) and the Office of Naval Research (N000140910589). The authors are grateful for the helpful comments by Andrew Lorenc and the anonymous reviewers.

Edited by: Z. Toth

Reviewed by: A. Lorenc, M. Buehner, and three other anonymous referees

\section{References}

Bishop, C. H., Etherton, B. J., and Majumdar, S. J.: Adaptive Sampling with the ensemble transform Kalman filter, Part I: Theoretical Aspects, Mon. Weather Rev., 129, 420-436, 2001.

Fertig, E. J., Baek, S.-J., Hunt, B. R., Ott, E., Szunyogh, I., Aravequia, J. A., Kalnay, E., Li, H., and Liu, J.: Observation bias correction with an ensemble Kalman filter, Tellus, 61A, 210-226, 2008.

Harris, L. M. and Durran, D. R.: An Idealized Comparison of One-Way and Two-Way Grid Nesting, Mon. Weather Rev., 138, 2174-2187, 2010.

Huang, X. Y., Xiao, Q., Barker, D., Zhang, X., Michalakes, J., Huang, W., Henderson, T., Bray, J., Chen, Y., Ma, Z., Dudhia, J., Guo, Y., Zhang, X., Won, D., Lin, H., and Kuo, Y.: FourDimensional Variational Data Assimilation for WRF: Formulation and Preliminary Results., Mon. Weather Rev., 137, 299-314, 2009.

Hunt, B. R., Kalnay, E., Kostelich, E. J., Ott, E., Patil, D., Sauer, T., Szunyogh, I., Yorke, J., and Zimin, A.: Four-Dimensional Ensemble Kalman Filtering, Tellus, 56A, 273-277, 2004.

Hunt, B. R., Kostelich, E., and Sunyogh, I.: Efficient data assimilation for spatiotemporal chaos: a local ensemble transform kalman filter, Physica D, 236, 112-126, 2007.

Juang, H. M. H.: A Spectral Fully Compressible Nonhydrostatic Mesoscale Model in Hydrostatic Sigma Coordinates: Formulation and Preliminary Results, Meteorol. Atmos. Phys., 50, 75$88,1992$.

Juang, H. M. H.: The NCEP Mesosacle Spectral Model: A Revised Version of the Nonhydrostatic Regional Spectral Model, Mon. Weather Rev., 128, 2329-2362, 2000.

Juang, H. M. H. and Hong, S. Y.: Sensitivity of the NCEP Regional Spectral Model to Domain Size and Nesting Strategy, Mon. Weather Rev., 129, 2904-2922, 2001.

Juang, H. M. H. and Kanamitsu, M.: The NMC Nested Regional Spectral Model, Mon. Weather Rev., 122, 3-26, 1994. 
Juang, H. M. H., Hong, S. Y., and Kanamitsu, M.: The NCEP Regional Spectral Model: An Update, Bull. Am. Meteorol. Soc., 78, 2125-2143, 1997.

Kalnay, E.: Atmospheric modeling, data assimilation, and predictability, Cambridge University Press, Cambridge, 2002.

Lynch, P. and Huang, P. M.: Initialization of the HIRLAM model using a digital filter, Mon. Weather Rev., 120, 1019-1034, 1992.

Ott, E., Hunt, B. R., Szunyogh, I., Zimin, A. V., Kostelich, E. J., Corazza, M., Kalnay, E., Patil, D. J., and Yorke, J. A.: A Local Ensemble Kalman Filter for Atmospheric Data Assimilation, Tellus, 56A, 415-428, 2004.

Sela, J. G.: Spectral Modeling at the National Meteorological Center, Mon. Weather Rev., 108, 1279-1292, 1980.

Szunyogh, I., Toth, Z., R. E. Morss, S. J. M., Etherton, B. J., and Bishop, S. H.: The effect of targeted dropsond observations during the 1999 Winter Storm Reconnaissance program, Mon. Weather Rev., 128, 3520-3537, 2000.
Szunyogh, I., Kostelich, E. J., Gyarmati, G., Kalnay, E., Hunt, B. R., Ott, E., Satterfield, E., and Yorke, J. A.: A Local Ensemble Transform Kalman Filter Data Assimilation system for the NCEP Global Model, Tellus, 60A, 113-130, 2008.

Tippett, M. K., Anderson, J. L., Bishop, C. H., Hamill, T. M., and Whitaker, J. S.: Ensemble square-root filters, Mon. Weather Rev., 131, 1485-1490, 2003.

Torn, R. D., Hakim, G. J., and Snyder, C.: Boundary Conditions fro limited-area ensemble Kalman filters, Mon. Weather Rev., 134, 2490-2502, 2006.

Warner, T. T., Peterson, R. A., and Treadon, R. E.: A tutorial on Lateral Boundary Conditions as a basic and Potentially Serious Limitation to Regional Numerical Weather Prediction, Bull. Am. Meteorol. Soc., 78, 2599-2617, 1997.

Zhang, F., Meng, Z., and Aksoy, A.: Tests of an Ensemble Kalman Filter for Mesoscale and Regional-scale Data Assimilation, Part I: Perfect Model Experiments, Mon. Weather Rev., 134, 722736, 2006 